\title{
A Command List Based Automatic Controlling Method of UAV for Infrastructure Monitoring System with Zero-standby Sensor Node
}

\author{
Yuta Suzuki, Shogo Yoshikawa, and Akira Yamawaki* \\ Kyushu Institute of Technology, 1-1 Sensui, Tobata, Kitakyushu 804-8550, Japan \\ *Corresponding Author: yama@ecs.kyutech.ac.jp
}

\begin{abstract}
Japan has been aging society and it will be going forward. The infrastructures also built during Japan's high economic growth period from 1960th to 1980th have also been aging. To maintain these infrastructures, many manpower will be required than current status. The combination of the wireless sensor network and UAV like drone is one of the technologies to reduce the monitoring cost of the infrastructures. The drone automatically gathers the sensing data from the sensor nodes attached to the many parts of the infrastructure. Using sensor network, it is desired that the sensor nodes attached to the infrastructure are powered by battery. This is because the sensor nodes can be equipped to the existing infrastructure easily and cheaply. However, the batter life becomes serious problem since the battery replacement to many sensor nodes may become to new burden to spend much manpower. Thus, we propose new infrastructure monitoring method combining the drone and the zero-standby power wireless sensor nodes. This paper develops a prototype of the command list based drone controller. Since this controlling method is independent to the GPS, we can use the drone at the places that cannot use the GPS, like the tunnel, under the bridges, shadow in the building, and so on.
\end{abstract}

Keywords: infrastructure, monitoring, sensor node, drone, battery, standby, aging society

\section{Introduction}

Japan has been aging society and it will be going forward. The infrastructures built during Japan's high economic growth period from 1960th to 1980th have also been aging. To maintain these infrastructures, many manpower will be required than current status. Whether enough manpower will be prepared or not is worried about in the aging society.

The usage of UAV like drone is one of the technologies to reduce the monitoring cost of the infrastructures ${ }^{(1-3)}$. By combining the wireless sensor network with the drone, more efficient infrastructure monitoring system can be realized. The drone automatically gathers the sensing data from the sensor nodes attached to the many parts of the infrastructure.

Using sensor network, it is desired that the sensor nodes attached to the infrastructure are powered by battery. This is because the sensor nodes can be equipped to the existing infrastructures easily and cheaply. However, the batter life becomes serious problem since the battery replacement to many sensor nodes may become to new burden spending much manpower. Thus, we propose new infrastructure monitoring method combining the drone and the zerostandby power wireless sensor nodes ${ }^{(4-6)}$.

This paper develops a prototype of the command list based drone controller. Since this controlling method is independent to the GPS, we can use the drone at the places that cannot use the GPS, like the tunnel, under the bridges, shadow in the building, and so on.

The rest of paper is organized as follows. Section 2 describes a conceptual overview of proposed infrastructure monitoring system combining with a drone and our zerostandby wireless sensor node. Section 3 shows the overview of the command list based drone controller. Section 4 describes the prototype controller to verify our proposal can be realized by using only the JavaScript package, Nodebebop ${ }^{(7)}$ on Node.js ${ }^{(8)}$ to make further development easy. Then, the preliminary experiments to verify the functionality of prototype controller are performed. Finally, Section 5 concludes this paper and indicates the future work. 


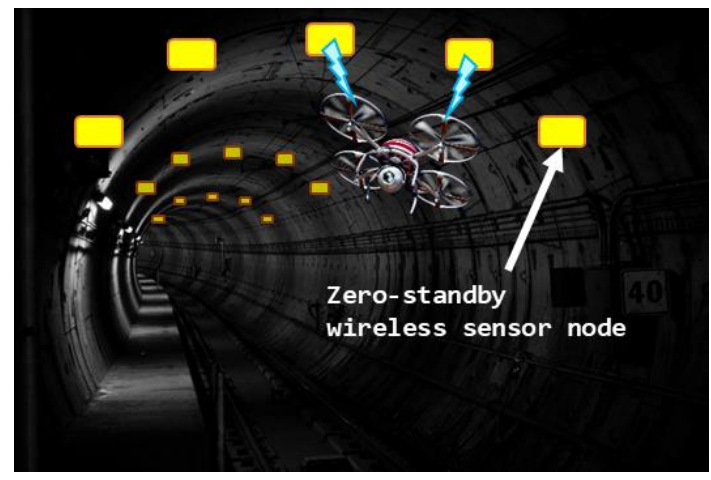

(a) Tunnel Monitoring

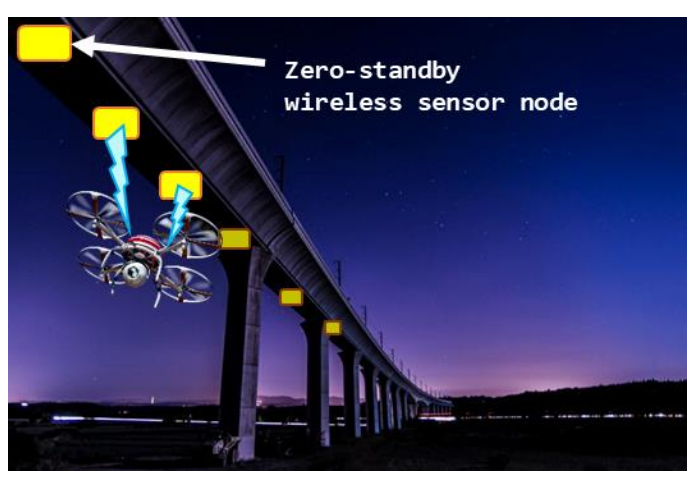

(b) Bridge Monitoring

Fig. 1. Proposed Infrastructure Monitoring System

\section{Proposed Monitoring System}

\subsection{Conceptual Overview}

Fig. 1 depicts a conceptual overview of the proposed infrastructure monitoring system combining a drone with our zero-standby wireless sensor nodes. In the proposed system, the drone used automatically flights following the command list previously made while invoking the dead sensor node, Zero-standby wireless sensor node, and gathering the sensor data sent from the invoked sensor node.

To make drones automatically flight, almost cases have employed the GPS and some map. However, the GPS cannot use at any places. For example, as shown in Fig. 1, the GPS cannot be used in the tunnel, under the bridge, shadow of high-building and so on. Thus, we attempt to realize the automatic flight of the drone by using the command list. The command list consists of the sequence of the drone actions. The command list is made from a 3D map of the infrastructure.

Sensor nodes attached to the infrastructures are preferred to employ the battery as the power supply. The battery life is significant to reduce the battery replacement cost. The zero-standby sensor node, the ZSSN we have

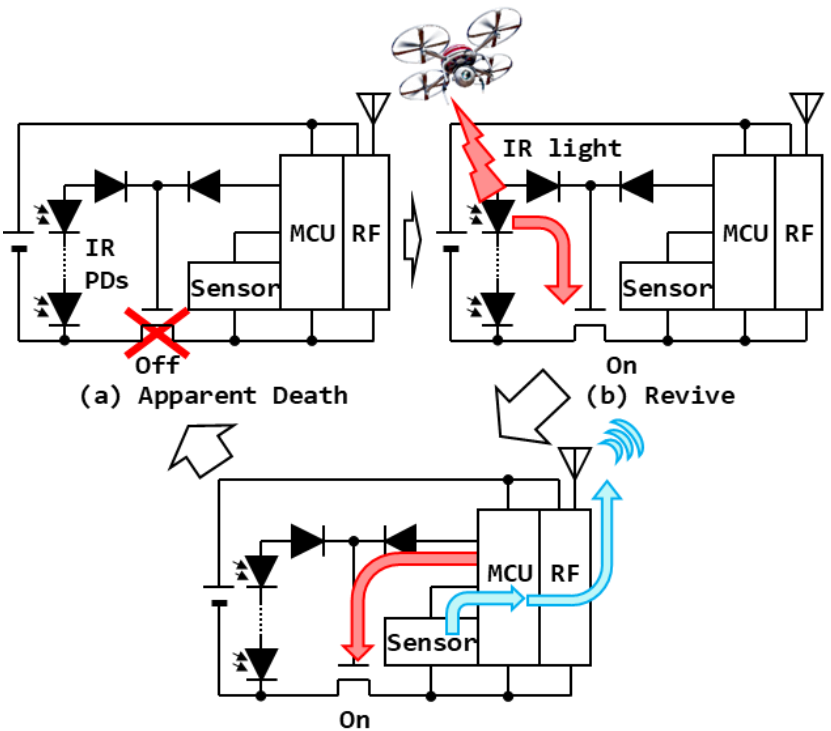

(c) Active

Fig. 2. Zero-standby Wireless Sensor Node

proposed is one of the candidates to make the battery life longer. This is because the standby power is completely reduced to zero. Since the standby power becomes zero, the dynamic power during execution consumes the battery power. In our monitoring system, the drone flights closer to the ZSSN at apparent death mode, revives it and acquires the sensing data via very near distance wireless communication with very weak RF. Thus, the power due to wireless communication is very weak. This leads to the lower dynamic power and the battery consumption. As a result, the battery life of the ZSSN may become longer than the convention sensor node due to the zero-standby and weak wireless communication.

As another effect of the ZSSN, we attempt to realize the uniform interface to any drone via the ZSSN hiding the differences over the sensors used. As a result, the drone has only to go close to the ZSSN without consideration of the differences over the sensors which are different according to the target infrastructures.

In the tunnels, the sunshine never comes to sensor nodes. The monitoring using the drone may also be often performed in night for safety reasons. In these cases, the solar cells cannot be used. So, we think that our zero-standby sensor node is useful in such cases.

\subsection{Zero-Standby Wireless Sensor Node}

Fig. 2 depicts the organization of the zero-standby wireless sensor node and its actions.

In the apparent death mode as shown in Fig. 2 (a), the sensor node does not consume the battery power because the ground line is cut by an N-ch MOSFET. 


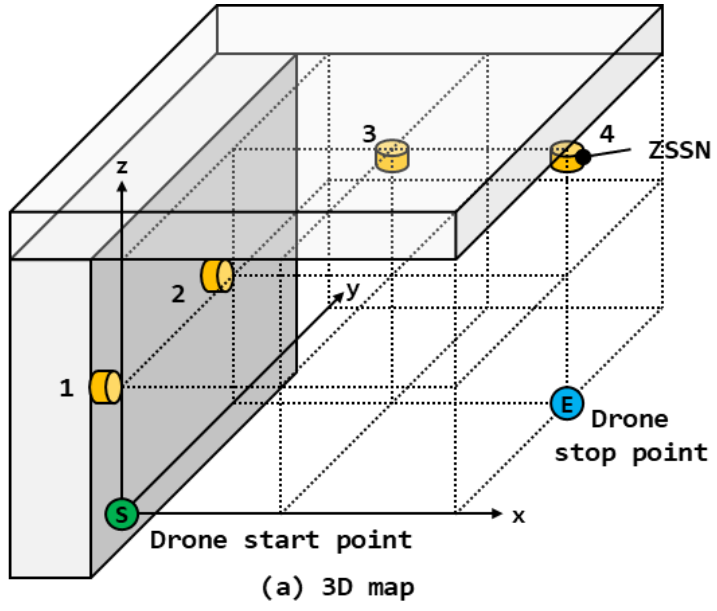

(a) 3D map

\begin{tabular}{ll} 
1: $\operatorname{start}() ;$ & $/ /$ Init. drone and take off \\
2: $\operatorname{move}(\theta, \theta, 1) ;$ & $/ /$ move $(x, y, z)$, move to $z+1$ \\
3: $\operatorname{align}(1) ;$ & $/ /$ drone alignment \\
4: $\operatorname{acquire}(1) ;$ & $/ /$ sensor data acquire \\
5: $\operatorname{move}(\theta, 1, \theta) ;$ & $/ /$ move to $y+1$ \\
6: $\operatorname{align}(2) ;$ & $/ /$ drone alignment \\
7: $\operatorname{acquire}(2) ;$ & $/ /$ sensor data acquire \\
8: $\operatorname{move}(1, \theta, \theta) ;$ & $/ /$ move to $x+1$ \\
9: $\operatorname{move}(\theta, \theta, 1) ;$ & $/ /$ move to $z+1$ \\
10: $\operatorname{align}(3) ;$ & $/ /$ drone alignment \\
11: $\operatorname{acquire}(3) ;$ & $/ /$ sensor data acquire \\
12: $\operatorname{move}(1, \theta, \theta) ;$ & $/ /$ move to $x+1$ \\
$13: \operatorname{align}(4) ;$ & $/ /$ drone alignment \\
14: $\operatorname{acquire}(4) ;$ & $/ /$ sensor data acquire \\
$15: \operatorname{move}(\theta, \theta,-1) ;$ & $/ /$ move to $z-1$ \\
$16: \operatorname{move}(\theta, \theta,-1) ; / /$ move to $z-1$ \\
17: $\operatorname{stop}() ;$ & $/ /$ Rounding and term. drone \\
\hline
\end{tabular}

(b) Command list

Fig. 3. Command List Based Drone Controller

The apparent death node is revived by the drone as shown in Fig. 2 (b). The drone irradiates the infrared light to IR photodiodes. The photodiodes exposed to the infrared light generate some electromotive force. The generated electromotive force drives the Nch MOSFET disconnecting the ground line. The driven MOSFET connects the ground line. As a result, the sensor node is revived and starts running.

The revived sensor node drives the Nch MOSFET instead of the photodiodes and goes to the active state as shown in Fig. 2 (c). The microcontroller, MCU, acquires the sensing data from the sensor used. The MCU processes the sensed data appropriately and sends it to the wireless module used. After such execution, the MCU stops driving the Nch MOSFET. As a result, the active sensor node goes to the apparent death mode naturally.

\section{Drone Controller Based on Command List}

To make an automatic drone flight independent to the GPS, we attempt to develop the command list based drone controlling method. The conceptual overview of our proposal is shown in Fig. 3.

A 3D map describing the target infrastructure has to be previously made as shown Fig. 3 (a). The 3D map has the coordinate of the start point where the drone is initially put to. The stop point as the final destination for the drone automatically flying is also included in the 3D map. The zero-standby wireless sensor nodes, ZSSN, are put to the coordinates so as to be attached to the target infrastructure.

We have to describe the command list so that the drone automatically flights along by ZSSNs from the start point to stop point. Fig. 3 (b) is an example of the command list for Fig. 3 (a). The drone analyzes the command list sequentially and performs the action specified by each command. At first, the drone initializes its own and takes off by start ( ). Then, the drone ascends toward to $Z$ axis by 1 unit due to move $(0,0,1)$. The drone may reach in front of the ZSSN \#1. However, the placement is shifted by several kinds of situations like wind, atmosphere, errors, and so on. Thus, the align (1) in the command list has the drone align to the ZSSN \#1 by the image processing via the embedded camera and a distance sensor to the target infrastructure. After such alignment, the drone revives the ZSSN \#1 and acquires the sensor data from the ZSSN \#1 by the acquire (1). To the stop point, the drone continuously performs the actions described in the command list as mentioned above, while performing the alignments to ZSSNs.

\section{Experiment and Discussion}

\subsection{Objective of Experiment}

As developing the proposed drone controlling method, we employ Node-bebop ${ }^{(7)}$ which is a package of Node.js ${ }^{(8)}$ to control Parrot bebop2 drone ${ }^{(9)}$. This is because the Nodebebop hides the detail of the bebop 2 drone by several APIs. That is, we think that the uniform command list to control the drone can be developed easily by using these APIs.

To develop the full command list, several aspects about APIs provided by Node-bebop must be investigated. This paper investigates following aspects.

The Node-bebop has APIs to move the drone like forward, backward, left, right, up, and down. These APIs has the input value ranging from 0 to 100 . We have to know how much the input value is needed to make the drone move by 1 unit on the 3D map. In addition, the duration time taken by moving 1 unit has to be known to estimate the traveling time of the drone. This is because we have to take care of the battery life about the flying drone and decide how many 
Tab. 1. Input value and duration time for moving $500 \mathrm{~mm}$ as 1 unit

\begin{tabular}{l|c|c}
\hline Direction & Input Value & Duration time \\
\hline Forward & 40 & $500 \mathrm{~ms}$ \\
Backward & 50 & $500 \mathrm{~ms}$ \\
Right & 45 & $640 \mathrm{~ms}$ \\
Left & 40 & $640 \mathrm{~ms}$ \\
\hline
\end{tabular}

Tab. 2. Output of Altimeter and Real Height

\begin{tabular}{c|c}
\hline Altimeter & Real Height \\
\hline 0.985 & $1.00 \mathrm{~m}$ \\
1.474 & $1.50 \mathrm{~m}$ \\
\hline
\end{tabular}

drones must be used according to the total monitoring time.

This experiment sets a unit of the 3D map to $500 \mathrm{~mm}$.

\subsection{Experimental Result and Discussion}

Tab. 1 shows the input values to the APIs provided by Node-bebop and the duration times taken to move $500 \mathrm{~mm}$ as 1 unit of 3D map. For the up and down, the meaning relationship between the input value and 1 unit could not be obtained. So, we eliminate these results and performs other experiment later. The shown actions via the APIs need the input values ranging from 40 to 50 . Their duration times range from 500 to $640 \mathrm{~ms}$. The measured values tended to be stable if without the external factors like wind, air flow reflected by some objects, and so on.

For up and down actions, we investigate another method using the altimeter which the bebop2 drone equips. Fig. 2 shows the relationship between the real height and the output value from the altimeter. The altimeter returns almost correct outputs corresponding to the real height by metric system. Due to the height limitation of the place where we performed the experiment, we were not able to investigate more than $1.5 \mathrm{~m}$ height. By using an altimeter supporting long range, some accurate height may be obtained at higher place.

Through those experiments mentioned above, we expect that some deviation to the zero-standby sensor node can be compensated by some align method. The traveling time also can be estimated relatively correct.

\section{Conclusions}

To develop new infrastructure monitoring system combining the drone with the zero-standby wireless sensor node, we have attempted to develop the command list based automatic drone flight method. For using Node-bebop as the medium to realize the uniform command list, we have investigated relationship among the input value of APIs provided by Node-bebop, the drone moving distance, and the duration time. The experimental results indicate some prospect that our proposal can be implemented practically.

As future work, we will develop the full command list based controller while performing the experiments which are closed to the practical environments.

\section{Acknowledgement}

This work was supported by JSPS KAKENHI Grant Number 17K00126.

\section{References}

(1) S. Sankarasrinivasan, E. Balasubramanian, K. Karthik, U. Chandrasekar, and Rishi Gupta, "Health Monitoring of Civil Structures with Integrated UAV and Image Processing System", Procedia Computer Science, Vol. 54, pp. 508-515, 2015.

(2) Youngjib Ham, Kevin K. Han, Jacob J Lin and M ani Golparvar-Fard, "Visual monitoring of civil infr astructure systems via camera-equipped Unmanned Aerial Vehicles (UAVs): a review of related work s", Visualization in Engineering, Vol. 4, No. 1, D OI 10.1186/s40327-015-0029-z, pp. 1-8, 2016.

(3) A. Ellenberg, A. Kontsos, F. Moon, and I. Bartoli, "Bridge related damage quantification using unmanned aerial vehicle", Struct. Control Health Monit. 2016; 23:1168-1179, 2016.

(4) Akira Yamawaki and Seiichi Serikawa: "Door Monitoring System Using Sensor Node with Zero Standby Power", Transactions on Engineering Technologies: International Multi-Conference of Engineers and Computer Scientists 2016, pp.73-87, 2017.

(5) Akira Yamawaki, Mayu Yamanaka and Seiichi Serikawa: "A sensor node architecture with zero standby power on wireless sensor network", Artificial Life and Robotics, vol. 20, no. 3, pp.210-216, 2015

(6) Akira Yamawaki and Seiichi Serikawa: "Battery Life Estimation of Sensor Node with Zero Standby Power Consumption", Proceedings of 19th IEEE International Conference on Computational Science and Engineering, pp.166-172, 2016.

(7) Node-bebop, https://github.com/hybridgroup/nodebebop [Accessed: 28- Feb-2018]

(8) Node.js, https://nodejs.org/ [Accessed: 28- Feb-2018]

(9) Parrot, https://www.parrot.com/ [Accessed: 28-Feb2018] 